\title{
INTERPLANETARY MISSIONS WITH THE GDM PROPULSION SYSTEM
}

\author{
T. Kammash \\ Department of Nuclear Engineering and \\ Radiological Sciences \\ The University of Michigan \\ Ann Arbor, Michigan 48109
}

(313) 764-0205

\author{
W. Emrich, Jr. \\ Marshall Space Flight Center \\ Huntsville, AL 35812 \\ (205) 544-7504
}

\begin{abstract}
The Gasdynamic Mirror (GDM) fusion propulsion system utilizes a magnetic mirror machine in which a hot dense plasma is confined long enough to produce fusion energy while allowing a fraction of its charged particle population to escape from one end to generate thrust. The particlcs escaping through the opposite end have their energy converted to electric power which can be used to sustain the system in a steady state operation. With the aid of a power flow diagram the minimum demands on energy production can be established and the propulsive capability of the system can be determined by solving an appropriate set of governing equations. We apply these results to several missions within the solar system and compute the trip time by invoking a continuous burn, acceleration/deceleration type of trajectory with constant thrust and specific impulse. Ignoring gravitational effects of the planets or the sun, and neglecting the change in the Earth's position during the flight we compute the round trip time for missions from Earth to Mars, Jupiter, and Pluto using linear distances and certain payload fractions. We find that a round trip to Mars with the GDM rocket takes about 170 days while those to Jupiter and Pluto take 494 and 1566 days respectively.
\end{abstract}

\section{INTRODUCTION AND BASIC PRINCIPLES}

Great interest has developed, since the Space Exploration Initiative was announced several years ago, in human exploration of the solar system and beyond. The major obstacle to such missions resides in the non-availability of propulsion systems that can allow them to take place in relatively short times so as to minimize exposure to dangerous galactic radiation. Although several schemes have been proposed in recent years none seem to be sufficiently advanced to be developed for deployment in the early part of the next century. The exception perhaps is with a fusion propulsion concept whose underlying physics is well established and the technology required for its construction is either currently available or will be so in the near future.

The device in question shown in Fig. 1 is the gasdynamic (GDM) mirror fusion propulsion system which is based on the simple magnetic mirror configuration that had been studied for many years as part of the fusion power research program. Unlike this machine, however, the plasma in GDM will be of such density and temperature as to make the ion-ion collision mean free path, $\lambda$, much shorter than its length, $L$. Under these conditions the plasma behaves like a fluid and its escape form the device is analogous to the flow of a gas into vacuum from a vessel with a hole as dictated by gasdynamic laws (Kammash 1995). The particle confinement time in GDM can be derived form basic principles, and expressed by (Kammash 1997):

$$
\tau=\frac{R L}{v_{t h}},
$$

where $R$ is the magnetic mirror ratio seen by the plasma, and $v_{t h}$ the particle thermal velocity. This mirror ratio is related to the vacuum mirror $R_{o}$ by : 


$$
R=\frac{R_{o}}{\sqrt{1-\beta}}
$$

where $\beta$ is the ratio of the plasma pressure to the vacuum magnetic field pressure and represents the efficiency of such a field in confining the hop plasma. The propulsive performance of GDM can be deduced from a simple model (Kammash 1995) that assumes that the dynamics is determined solely by the ions, and that the electrons can be ignored except for the power they radiate from the system. The plasma in a high aspect ratio device can be shown to be stable against magnetohydrodynamic (MHD) and microinstabilities (Kammash 1998) which if allowed to arise or persist can destroy it's propulsive capability. Though somewhat simplified the model in question is sufficiently comprehensive to provide a meaningful assessment of GDM's capability for interplanetary travel in the solar system.

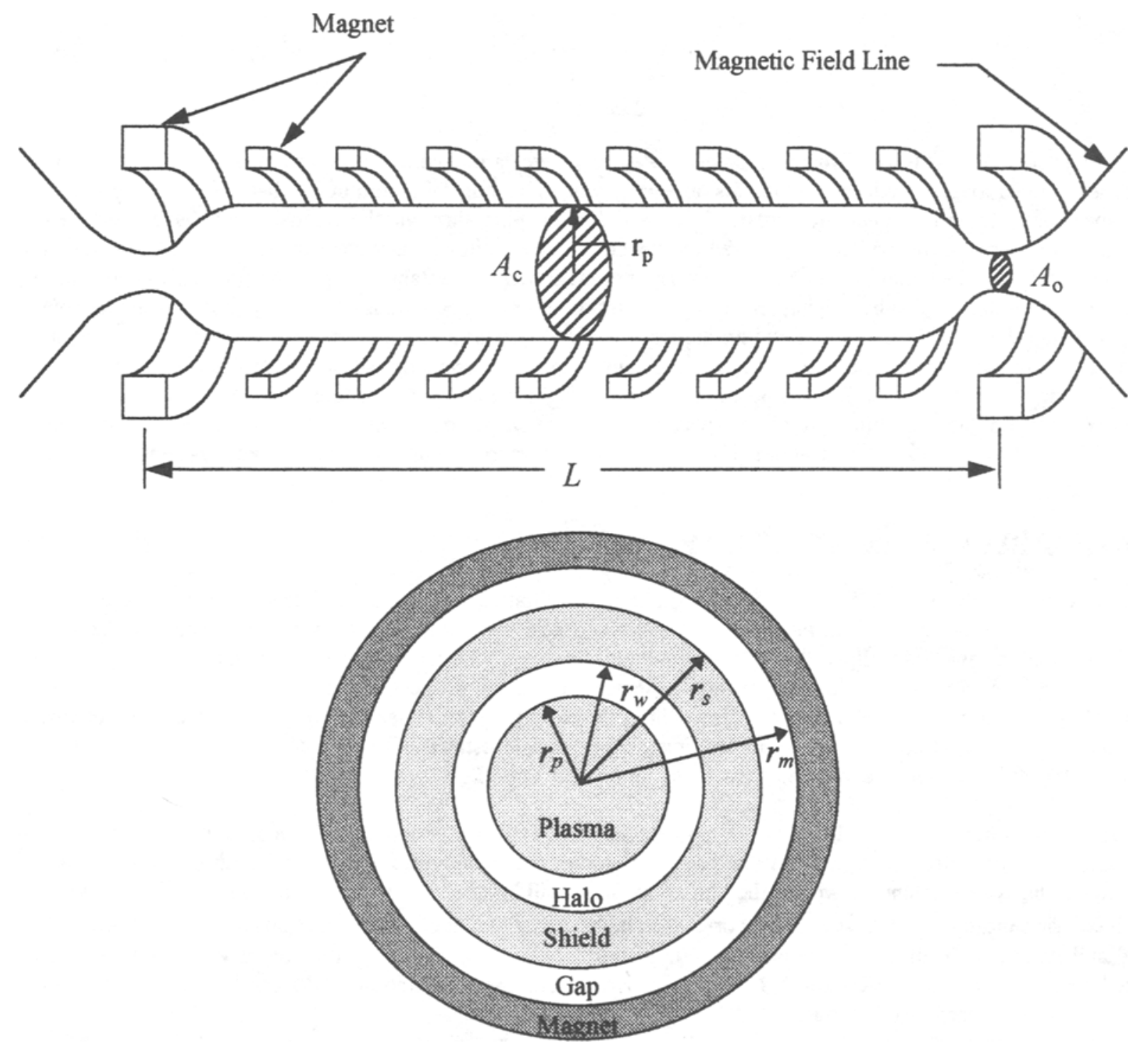

FIGURE 1. Schematic and Cross-Sectional View of the Gasdynamic Fusion Propulsion System. 


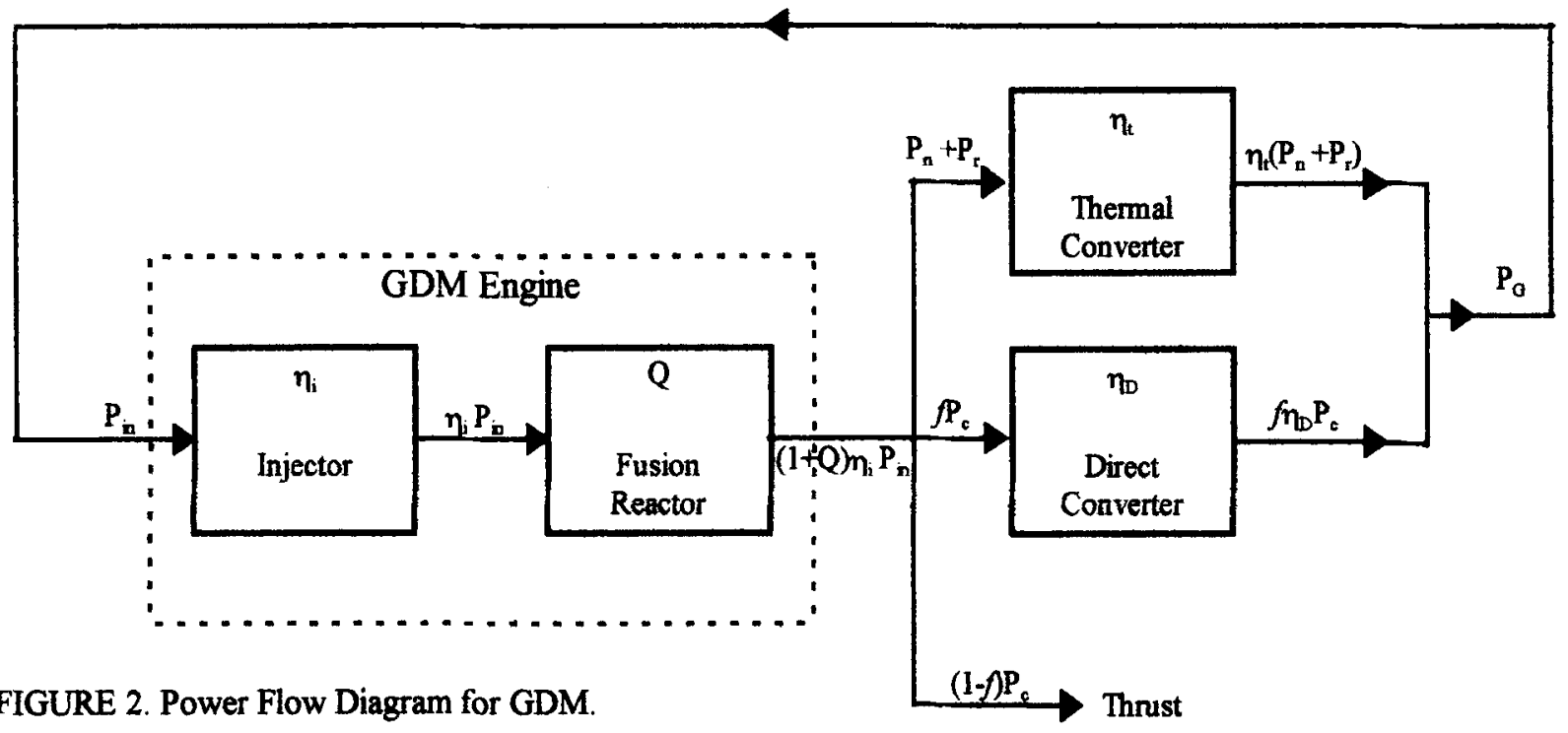

The reactor, which generates the fusion energy, and the injector which supplies power to the plasma to heat it to thermonuclear temperatures, constitute the GDM engine which along with other components make up the total propulsion system. This is reflected in the power flow diagram shown in Fig. 2 which reveals a thermal converter that processes at an efficiency $\eta_{i}$ the neutron power $P_{n}$, and the radiated power $P_{r}$ which is the sum of the Bremsstrahlung power, $P_{B}$, and the synchrotron radiation power $P_{s}$. It also shows a direct converter which converts to electricity at an efficiency $\eta_{D}$ the power of the charged particles that pass through one end of GDM to that component. If we designate by $f$ the fraction of these charged particles that go to the direct converter then $(1-f)$ emerge through the opposite mirror to generate the thrust. If the gross electric power $P_{G}$ is set exactly equal to the power going to the injector then a critical energy multiplication factor $Q_{c}$ can be calculated that makes the system self-supporting. It is given by :

$$
Q_{c}=\frac{1-f \eta_{i} \eta_{D}}{f \eta_{i} \eta_{D}-\frac{\left(P_{n}+P_{r}\right)}{P_{f}} \eta_{i}\left(f \eta_{D}-\eta_{t}\right)}
$$

where $P_{f}$ is the fusion power and $\eta_{i}$ the injector efficiency generally taken as unity. For a symmetric mirror, i.e. $f=1 / 2$ and $\eta_{t}=1 / 2 \eta_{D}$, Eq. (3) shows that the critical value of $Q$ is independent of the fuel cycle. In fact for $\eta_{D}=0.9$ and $\eta_{t}=0.45, Q_{c}$ has a value of 1.222 which is quite modest and easily achievable compared to the large $Q$-values required by terrestrial power reactors.

We employ the above model in calculating the propulsion performance of two systems: one using a deuterium-tritium (DT) fuel cycle and another using deuterium-helium ${ }^{3}$ (D-He ${ }^{3}$ ). The plasma and propulsion parameters for these systems are given in Table 1 . The most striking difference between the two cases is the operating temperature and correspondingly the radiative losses. Because of the higher temperature of the plasma is much larger in the $\mathrm{D}-\mathrm{He}^{3}$ case requiring a larger magnetic field for confinement, which in turn leads to a larger vehicle mass. 
TABLE 1. GDM Plasma and Propulsion Parameters.

\begin{tabular}{|l|c|c|}
\hline \multicolumn{2}{|c|}{$\beta=0.95, \mathrm{Q}=1.222, \mathrm{R}=100$} \\
\hline Parameter & $\mathbf{D}-\mathrm{T}$ & $\mathbf{D}-\mathrm{He}^{3}$ \\
\hline Plasma Density $\left(\mathrm{cm}^{-3}\right)$ & $1.0 \times 10^{16}$ & $1.0 \times 10^{16}$ \\
\hline Plasma Temperature (keV) & 10 & 60 \\
\hline Plasma Radius (cm) & 5 & 5 \\
\hline Plasma Length (m) & 44 & 1297 \\
\hline Confinement Time (s) & $4.07 \times 10^{-3}$ & $4.92 \times 10^{-2}$ \\
\hline Central Magnetic Field (Tesla) & 9.21 & 24.73 \\
\hline Fusion Power (MW) & $2.730 \times 10^{3}$ & $5.675 \times 10^{4}$ \\
\hline Bremsstrahlung Power (MW) & 58.17 & $1.703 \times 10^{4}$ \\
\hline Synchrotron Radiation Power (MW) & 18.94 & $4.205 \times 10^{4}$ \\
\hline Neutron Power (MW) & $2.183 \times 10^{3}$ & $6.213 \times 10^{3}$ \\
\hline Thrust (N) & $2.512 \times 10^{3}$ & $1.437 \times 10^{4}$ \\
\hline Thrust Power (MW) & $1.351 \times 10^{3}$ & $1.894 \times 10^{4}$ \\
\hline Injection Power (MW) & $4.643 \times 10^{4}$ \\
\hline Engine Mass (Mg) & $2.233 \times 10^{3}$ & 3015 \\
\hline Total Vehicle Mass (Mg) & 101 & 4434 \\
\hline Specific Power (kW/kg) & 422 & 6.28 \\
\hline Specific Impulse (s) & 13.40 & $3.106 \times 10^{3}$ \\
\hline Mars Round Trip (days) & $1.268 \times 10^{5}$ & 228 \\
\hline
\end{tabular}

\section{MISSION ANALYSIS}

In Table 1, we included the example of a round trip mission to Mars as a measure of the propulsive capability of the system. In obtaining the travel time we employed a constant thrust, constant specific impulse continuous burn, acceleration/deceleration type of trajectory for which the round trop time $\tau_{R T}$ can be expressed by :

$$
\tau_{R T}=\frac{4 l}{g I_{s p}}+4 \sqrt{\frac{l m_{f}}{F}}
$$

where $l$ is the linear distance between the planets, $g$ the Earth's gravitational acceleration, $I_{s p}$ the specific impulse, $m_{f}$ the final (dry) mass of the vehicle and $F$ the thrust. Eq. (4) neglects the effect of gravity of the planets involved as well as that of the sun, and also ignores the change in the Earth's position during the flight. Although the D-He ${ }^{3}$ fueled system generates larger propulsion parameters that the DT fueled system it results in a $35 \%$ increase in travel time due to the significantly larger vehicle mass. We have examined other missions in the solar system including one to the outermost planet, Pluto. The results are given in Table 2 for a DT burning GDM rocket. 
TABLE 2. Solar System Missions with GDM.

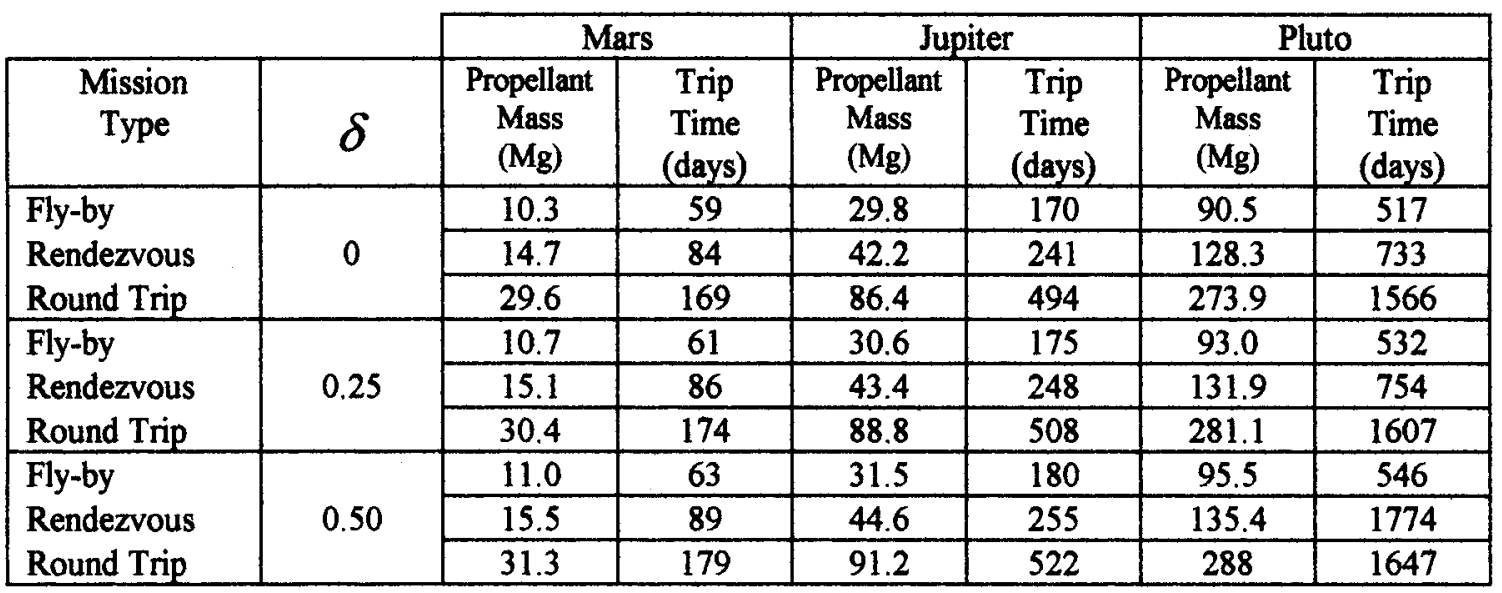

\section{SUMMARY}

Table 2 gives the results for three types of missions from Earth to the planets shown for various payloads: Fly-by where the vehicle simply passes the planet, a Rendezvous where the vehicle ends up circling the planet, and the round trip where the vehicle returns to Earth following a trajectory similar to that of the outbound journey. In all cases enough propellant (fuel) is carried on board to just fulfill the mission. The most striking feature of GDM's performance as a propulsion system is the small amount of fuel required to achieve these missions. We see, for example, that the propellant mass for a Mars round trip with about $50 \mathrm{Mg}$ payload is about $7 \%$ of the total vehicle mass, while in the case of a round trip to Jupiter at $25 \%$ payload is about $20 \%$ of the total vehicle mass. In short, the propellant mass required for round trip missions in the solar system with GSM may be significantly smaller than the dry mass of the vehicle, where as it may be many times larger in the case of chemical or even nuclear thermal propulsion systems. It is clear, therefore, why the gasdynamic mirror fusion propulsion system might make an especially attractive propulsion device for solar system exploration and beyond when one of the side benefits is effective removal of launch window limitations.

\section{Acknowledgments}

This work was supported by NASA.

\section{References}

Kammash, T., and M. J. Lee (1995) "Gasdynamic Fusion Propulsion System for Space Exploration, "Journal of Propulsion and Power, 11:544-553.

Kammash, T., M. J. Lee and D. I. Poston (1997) "High Thrust High Specific Impulse Gasdynamic Fusion Propulsion System," Journal of Propulsion and Power, 13:421-427.

Kammash, T., and W. Emrich (1998) "Physics Basis for the Gasdynamic Mirror (GDM) Fusion Rocket," STAIF-98.

Nomenclature

$F: \quad$ Thrust $(\mathrm{N})$

1: $\quad$ Linear Distance Between Planets (km)

g: Gravitational Acceleration $\left(\mathrm{m} / \mathrm{s}^{2}\right)$

$I_{s p}: \quad$ Specific Impulse (s)

$m_{f}$ : Dry Mass of Vehicle $(\mathrm{Mg})$
$P_{f}: \quad$ Fusion Power (MW)

$P_{n}: \quad$ Neutron Power (MW)

$P_{r}$ : $\quad$ Radiated Power (MW)

$P_{s}: \quad$ Synchrotron Radiation Power (MW)

$P_{B}$ : Bremsstrahlung Power (MW) 
$P_{G}: \quad$ Gross Electric Power (MW)

$f: \quad$ Fraction of Particles to Direct Converter

$Q: \quad$ Fusion Energy Multiplication

$Q_{c}: \quad$ Critical Q-Value

$\tau$ : Plasma Confinement Time (s)

$\tau_{R T}: \quad$ Round Trip Time (days)

$\eta_{i}: \quad$ Injector Efficiency

$\eta_{t}$ : Thermal Converter Efficiency
$\eta_{D}: \quad$ Direct Converter Efficiency

$L: \quad$ Plasma Length (m)

$R$ : $\quad$ Plasma Mirror Ratio

$R_{0}$ : Vacuum Mirror Ratio

$v_{t h}:$ Ion Thermal Velocity $(\mathrm{m} / \mathrm{s})$

$\beta$ : $\quad$ Ratio of Plasma Pressure to Magnetic Field Pressure

$\delta: \quad$ Engine Mass / Payload Mass

2. Mean Eree Path (m) 